4 the challenge and adapted. This oil painting of Abi Schofield (Fig. 3), one of the dental nurses at Sandy Lane, was done to reflect the new ways of working including surgery fallow time following aerosol generating procedures (now known as post AGP downtime).

Denise said that she felt it was time that the team at Sandy Lane Dental Practice and all UK dental personnel were thanked for their continued professionalism and care in these unprecedented times.

As Lancashire continues to be in the highest Tier 3 restrictions, Denise recently did an oil painting to display at the practice (Fig. 4). The bouquet of flowers was painted as a tribute to the team's unwavering commitment to the continued provision of dental care in the area.

\section{Thank You}

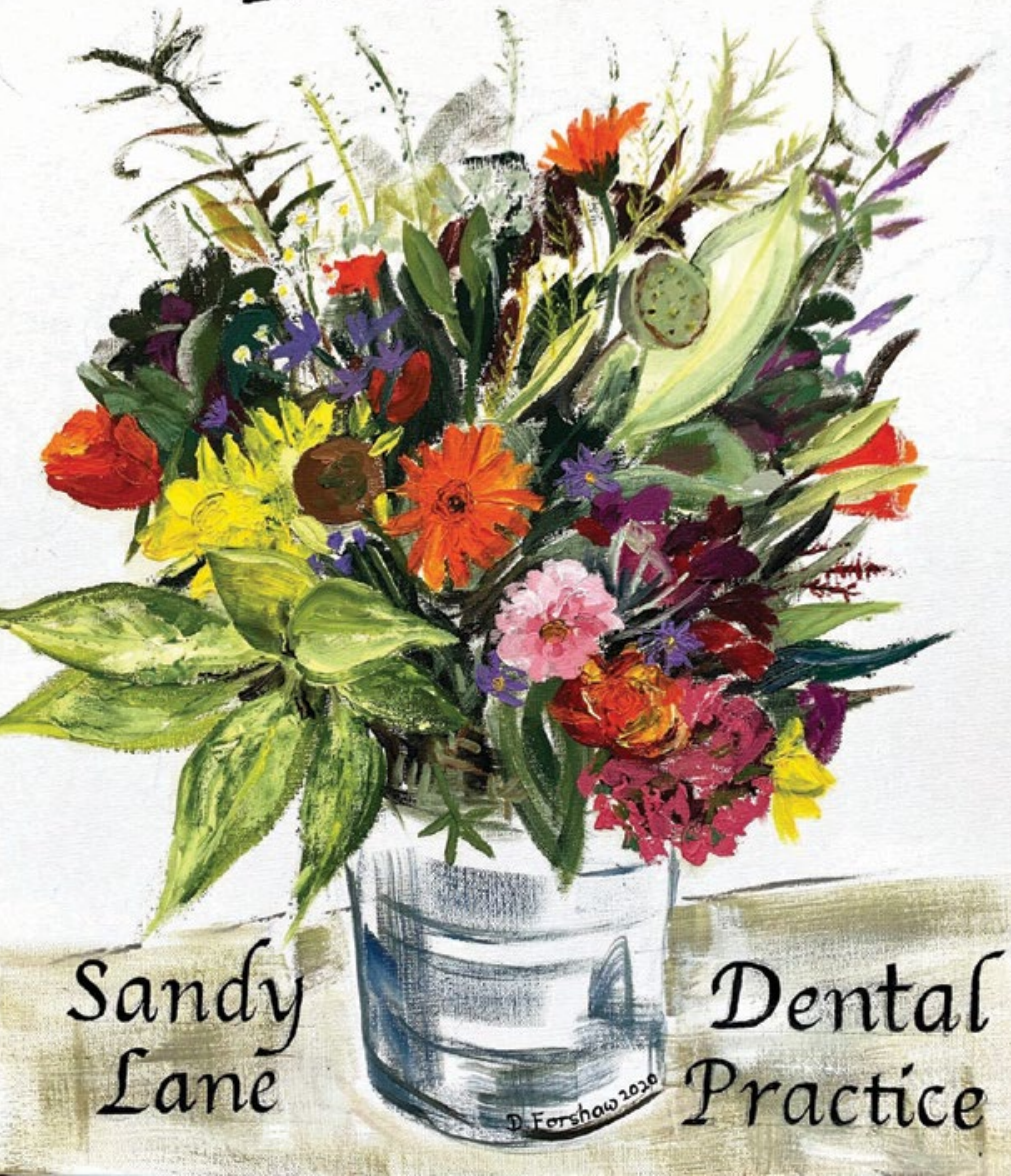

Fig. 4 An oil painting of a bouquet of flowers painted as a tribute to Sandy Lane

\section{Dental Showcase moves online for 2021}

The BDIA Dental Showcase Advisory Board and MA Exhibitions Limited have taken the decision to reschedule BDIA Dental Showcase to 25-26 March 2022 at ExCeL, London. For 2021, a brand new virtual event will take place on 22-26 March 2021: BDIA Dental Showcase On Demand.

For more information and to register your interest in the virtual event, visit https://ondemand.dentalshowcase.com/.

\section{Research grants available for those with a great idea}

Two grants of up to $£ 10,000$ are available to members of the British Society of Paediatric Dentistry (BSPD) looking for pumppriming funds for research projects focused on the oral health of children. The deadline for submission of an application for a joint FDS RCSEng BSPD grant is 29 January 2021.

In order to apply, you must be a member of BSPD and have developed a research project which relates to children's oral health in the broadest sense. Applicants do not have to be a member of the FDS RCSEng but would be expected to join for the duration of the grant.

In 2020, Laura Timms, an academic clinical fellow based at the University of Sheffield School of Dentistry, was successful in her application for pump-priming funds. She was awarded $£ 5,460$ to support background research into a video to be made by and for children, explaining the silver diamine fluoride technique.

Chris Vernazza, BSPD's academic lead, said: 'Having good quality evidence is key to the Society's mission of improving the oral health of children in the UK and so we are delighted to be working with RCS England's Faculty of Dental Surgery to support research. The grants offer an ideal opportunity to undertake a small project, hopefully leading on to further research and so we would encourage anyone with great ideas to apply'.

For more information visit https://www.rcseng.ac.uk/ dental-faculties/fds/research/fds-pump-priming-grants/\#fdsbspd.

\section{BDA Benevolent Fund sees three-fold} \section{increase in requests for help}

This year, the BDA Benevolent Fund has seen a threefold increase in requests for help, a direct result of the challenges posed by COVID-19, and has been able to maintain the usual high level of assistance thanks to the dedication of the team and robust protocols.

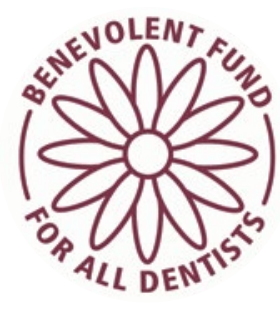

With Christmas fast approaching, General Manager, Laura Hannon, recognises that the strain may increase for some.

Laura said: 'We are here to help. Between now and the end of the year, we will be doing all we can to keep a roof over people's heads, as well as fed, safe and warm.

'We will also give people additional support so that they can feel a sense of normalcy, for example ensuring the children of beneficiaries do not miss out on a present, or that no-one spends Christmas Day alone because they cannot afford to travel to their family - if COVID19 rules allow that'.

If you, or someone you know, are in need, please contact the BDA Benevolent Fund straight away in confidence. Simply call 0207486 4994 or visit www.bdabenevolentfund.org.uk for more information. 